\title{
Robust Structure Preserving Nonnegative Matrix Factorization for Dimensionality Reduction
}

\author{
Bingfeng Li, ${ }^{1,2,3}$ Yandong Tang, ${ }^{1}$ and Zhi Han ${ }^{1}$ \\ ${ }^{1}$ State Key Laboratory of Robotics, Shenyang Institute of Automation, Chinese Academy of Sciences, Shenyang 110016, China \\ ${ }^{2}$ School of Electrical Engineering and Automation, Henan Polytechnic University, Jiaozuo 454000, China \\ ${ }^{3}$ University of Chinese Academy of Sciences, Beijing 100049, China \\ Correspondence should be addressed to Bingfeng Li; libingfeng@hpu.edu.cn
}

Received 25 January 2016; Revised 2 June 2016; Accepted 2 June 2016

Academic Editor: Wanquan Liu

Copyright (C) 2016 Bingfeng Li et al. This is an open access article distributed under the Creative Commons Attribution License, which permits unrestricted use, distribution, and reproduction in any medium, provided the original work is properly cited.

\begin{abstract}
As a linear dimensionality reduction method, nonnegative matrix factorization (NMF) has been widely used in many fields, such as machine learning and data mining. However, there are still two major drawbacks for NMF: (a) NMF can only perform semantic factorization in Euclidean space, and it fails to discover the intrinsic geometrical structure of high-dimensional data distribution. (b) NMF suffers from noisy data, which are commonly encountered in real-world applications. To address these issues, in this paper, we present a new robust structure preserving nonnegative matrix factorization (RSPNMF) framework. In RSPNMF, a local affinity graph and a distant repulsion graph are constructed to encode the geometrical information, and noisy data influence is alleviated by characterizing the data reconstruction term of NMF with $\ell_{2,1}$-norm instead of $\ell_{2}$-norm. With incorporation of the local and distant structure preservation regularization term into the robust NMF framework, our algorithm can discover a low-dimensional embedding subspace with the nature of structure preservation. RSPNMF is formulated as an optimization problem and solved by an effective iterative multiplicative update algorithm. Experimental results on some facial image datasets clustering show significant performance improvement of RSPNMF in comparison with the state-of-the-art algorithms.
\end{abstract}

\section{Introduction}

With the development of data acquisition technology, highdimensional data is ubiquitous in many areas. Directly learning in original data space is prone to disturbance by noise; meanwhile, it is hard to discover the latent semantic structure [1], so low-dimensional representation learning becomes a fundamental problem in numerous data analysis algorithm [2-10]. As a common used tool for dimensionality reduction, matrix factorization has received more and more attention in recent years [2, 3, 11]. Unlike typical matrix factorization methods, such as QR and SVD, which seek for exact factorization of original matrix, nonnegative matrix factorization (NMF) approximately factorizes the original matrix into two low-dimensional nonnegative submatrixes. It is the nonnegative constraint which assures NMF can learn a sparse and part-based representation of the sample matrix. Psychological and physiological evidences have proved that NMF is consistent with how the brain interprets an object in a part-based, nonsubtractive way [12-14]. Recently, NMF has exhibited great success in a variety of applications, like text analysis [15], image processing [16, 17], and video representation [18].

In order to detect the latent geometrical structure hidden in high-dimensional data space, many manifold learning algorithms have been proposed, for instance, isometric feature mapping (ISOMAP) [19], locally linear embedding (LLE) [20], Laplacian eigenmaps (LE) [21], and local tangent space alignment (LTSA) [22]. Motivated by the benefit of NMF and manifold learning, a graph regularized NMF (GNMF) method is proposed in [5]. Although GNMF displays some improvement on clustering performance, it suffer greatly from the following two problems in real-world applications: (a) GNMF only consider the ideal case where the input data is noise-free. When the input data samples is corrupted by noise, GNMF even leads to an inaccurate matrix factorization. (b) GNMF overemphasizes the affinity relationship 
between neighboring data samples; the repulsion relationship between data samples which is favorable for clustering is even neglected thoroughly.

To address the abovementioned issues of GNMF, in this paper, we propose a new robust structure preserving nonnegative matrix factorization (RSPNMF) method. In RSPNMF, the data reconstruction term is formulated in the form of robust $\ell_{2,1}$-norm instead of $\ell_{2}$-norm, which will improve the model robustness to noisy data; meanwhile, the rotation invariance property of the model is also preserved. The success of GNMF relies on the assumption that two neighboring data samples share the same semantic label; it characterizes the compactness relationship among neighboring data samples. Inspired by this observation, we propose a new assumption; that is, data samples which are remote are generally semantic difference; and then we constrain that the low-dimensional representation of distant data samples in the original data space should also be far apart in embedding subspace. We consider that the preservation of neighboring data sample's compactness together with distant data sample's repulsion is helpful to describe the intrinsic geometrical structure of data distribution.

The contributions of this paper are presented as follows:

(1) A novel nonnegative matrix factorization algorithm, named RSPNMF, is proposed, in which the intrinsic manifold structure is approximated with the local affinity and distant repulsion graphs. By incorporating the corresponding regularization term into robust NMF framework, our proposed algorithm can find the latent subspace of the original matrix with nature of manifold structure learning.

(2) We formulate our approach as an optimization problem and provide an iterative multiplicative update algorithm to solve it. The convergence proof of the proposed algorithm is also provided.

(3) Our experiments on several datasets for clustering validate our proposed algorithm. Compared with several state-of-the-art methods, the results of our RSPNMF show the better performance.

The rest of the paper is organized as follows. In Section 2, we briefly review the background of NMF and related works. In Section 3, our RSPNMF approach and the convergence proof of optimization scheme are presented. Some experimental results and comparison with other approach are presented in Section 4. We end this paper with some conclusions and discussion in Section 5.

\section{Related Works}

Given a nonnegative matrix $X=\left[x_{1}, \ldots, x_{N}\right], x_{i} \in \mathbb{R}_{+}^{M \times 1}$ is a data sample. NMF attempts to find two low-dimensional nonnegative submatrixes whose product provides a best approximation to the original matrix $X$; that is,

$$
\begin{array}{ll} 
& X \approx U V^{T} \\
\text { s.t. } & U \in \mathbb{R}_{+}^{M \times K}, \\
& V \in \mathbb{R}_{+}^{N \times K} .
\end{array}
$$

With the purpose of finding a compact factorization for the original data matrix, the number of basis component $K$ usually is set less than $M$ and $N$.

One common data reconstruction function used to quantify the approximation of NMF is formulated as follows:

$$
O=\left\|X-U V^{T}\right\|_{F}^{2}
$$

where $\|\cdot\|_{F}$ denotes the Frobenius norm.

Due to no-convexity of the objective function in (2) on both $U$ and $V$, it is unrealistic to expect an algorithm to find its global minimum. An iterative multiplicative update algorithm which can find the local minima of (2) is proposed in [23], which are

$$
\begin{aligned}
& u_{i k} \longleftarrow u_{i k} \frac{(X V)_{i k}}{\left(U V^{T} V\right)_{i k}}, \\
& v_{j k} \longleftarrow v_{j k} \frac{\left(X^{T} U\right)_{j k}}{\left(V U^{T} U\right)_{j k}} .
\end{aligned}
$$

As the update algorithm allows only additive combination among different basis components, it is believed that NMF can learn a sparse and part-based representation [4].

Inspired by the work exhibited in [23], many of NMF variants have been proposed over the past years. In order to explicitly impose sparseness constraint, sparseness constrained NMF is proposed in [9, 24-28]; these works expect more sparse representation than the original NMF. To characterize different residual error model, two new distance metrics are introduced in $[29,30]$. Similar to SVD presented in [31], a nonnegative matrix can also be factorized into three nonnegative components. In [32], a fast nonnegative matrix trifactorization is proposed, and then orthogonality constrained nonnegative matrix trifactorization is studied in [27]. Different from unsupervised NMF variants, a semisupervised nonnegative matrix decomposition algorithm is proposed in [9], where the label information of data samples was introduced to NMF as an additional constraint. To improve discriminating power of MMF, in [33, 34], Fisher's criterion as an additional regularization term is incorporated into NMF framework. In spite of the success of the abovementioned NMF variants in various scenarios, all of them perform matrix factorization on Euclidean space.

In order to integrate manifold learning into NMF framework, a graph regularized NMF (GNMF) algorithm is firstly proposed in [6]; then some GNMF variants are introduced in [35-38].

In $[39,40]$, a dual regularized seminonnegative matrix trifactorization is proposed, in which data samples and 
feature samples manifold structure are also considered. Although the graph-based NMF algorithm incorporates the prior knowledge of manifold structure into NMF framework, overemphasizing local structure preservation sometimes will degrade its performance. A structure preserving nonnegative matrix factorization (SPNMF) is proposed in [10], in which the intrinsic manifold is approximated with local and distant graphs. Experiment results demonstrate SPNMF outperforms GNMF and its variants.

All the abovementioned NMF variants are based on the premise that the input data are noise-free; it is impractical to real-world data samples which are often corrupted by noise. For boosting NMF robustness to outliers, $\ell_{2,1}$-norm based NMF reconstruction function is applied in [41, 42]. In [43], $\ell_{2,1}$-norm and $\ell_{1}$-norm are used to formulate the graph embedding and data reconstruction function, which endows the algorithm with robustness to unreliable graphs and noisy labels.

Among the existing methods, SPNMF is closely relevant to our method and performs better on some specific datasets. There are still some differences between our proposed algorithm and SPNMF. Firstly, the Laplacian matrix of distant repulsion graph from SPNMF needs to be updated in each iteration, which is time consuming. Additionally, to learn a better parts-based representation, the basis vector of SPNMF is required to be as orthogonal as possible. Finally, SPNMF is sensitive to noisy data points.

\section{The Proposed Method}

Our goal is to perform matrix factorization on the hidden semantic subspace. To this end, we impose the local affinity and distant repulsion structure preservation on the new robust NMF framework. In this section, we will first explain the structure preservation and robust data reconstruction terms of RSPNMF, followed by its optimization algorithm. The convergence of the proposed algorithm is proved finally.

3.1. Problem Formulation. Despite the diverse motivations of various dimensionality reduction algorithms, most of them can be explained within a graph embedding framework. Let $G=\{X, E\}$ be an undirected weighted graph, where vertex set $X$ corresponds to a dataset and $E \in \mathbb{R}_{+}^{N \times N}$ is an affinity matrix whose elements measure the similarity of each pair of vertices. In graph embedding algorithm, graph $G$ characterizes the prior knowledge of geometric structure from data distribution.

If we use the $k_{1}$-nearest neighboring graph $G$ to characterize the local structure of data distribution, the weight matrix $W=\left[w_{i j}\right]_{N \times N}$ can be defined as follows:

$$
\begin{aligned}
& w_{i j} \\
& = \begin{cases}e^{-\left\|x_{i}-x_{j}\right\|^{2} / \sigma^{2}}, & \text { if } x_{i} \in N_{k_{1}}\left(x_{j}\right) \text { or } x_{j} \in N_{k_{1}}\left(x_{i}\right), \\
0, & \text { otherwise, }\end{cases}
\end{aligned}
$$

where $\sigma$ is the bandwidth parameter and $N_{k_{1}}\left(x_{i}\right)$ denotes the set of $k_{1}$ nearest neighbors of $x_{i}$.
Local invariance assumption which encourages neighboring data pairs in the original space to be still close in the low-dimensional embedding subspace can be formulated as

$$
\min _{V} \frac{1}{2} \sum_{i, j=1}^{N}\left\|v_{i}-v_{j}\right\|^{2} w_{i j} \text {, }
$$

where $v_{i}$ is the corresponding low-dimensional representation of $x_{i}$.

By defining the diagonal matrix $D$ and Laplacian matrix $L$ of graph $G$ are as

$$
\begin{aligned}
L & =D-W \\
d_{i i} & =\sum_{i \neq j} w_{i j}, \quad \forall i
\end{aligned}
$$

we can rewrite (5) as

$$
\begin{aligned}
& \min _{V} \frac{1}{2} \sum_{i, j=1}^{N}\left\|v_{i}-v_{j}\right\|^{2} w_{i j} \\
& \quad=\min _{V}\left(\sum_{i=1}^{N} v_{i}^{T} v_{i} d_{i i}-\sum_{i, j=1}^{N} v_{i}^{T} v_{j} w_{i j}\right) \\
& =\min _{V}\left(\operatorname{Tr}\left(V^{T} D V\right)-\operatorname{Tr}\left(V^{T} W V\right)\right) \\
& \quad=\min _{V} \operatorname{Tr}\left(V^{T} L V\right),
\end{aligned}
$$

where $\operatorname{Tr}()$ denotes the trace of a matrix.

Equation (7) essentially holds the smoothness of dimensionality reduction process. It is based on two vital assumptions; firstly, the neighboring data samples in highdimensional space are semantic similarity, and secondly the preservation of affinity structure plays great role for low-dimensional representation.

Local invariance assumption essentially exploits the favorite relationship among similar data samples under unsupervised condition; however, it ignores unfavorite relationship between divergent data pairs. In this paper, we conjecture that the distant data pairs are always semantic difference. A new distant neighboring graph $G^{C}=\left\{X, E^{C}\right\}$ which is used to describe the repulsion relationship between dissimilar data pairs is also constructed. The corresponding weight matrix $W^{C}=\left[w_{i j}^{c}\right]_{N \times N}$ is defined as follows:

$$
\begin{aligned}
& w_{i j}^{c} \\
& = \begin{cases}e^{-\left\|x_{i}-x_{j}\right\|^{2} / \sigma^{2}}, & \text { if } x_{i} \in D_{k_{2}}\left(x_{j}\right) \text { or } x_{j} \in D_{k_{2}}\left(x_{i}\right), \\
1, & \text { otherwise, }\end{cases}
\end{aligned}
$$

where $D_{k_{2}}\left(x_{i}\right)$ denotes the remotest $k_{2}$ data samples of $x_{i}$ in givensample dataset.

From (8), we can we can find that the more the distance between $x_{i}$ and $x_{j}$, the smaller the value of $w_{i j}^{c}$. If we want the corresponding low-dimensional representation $v_{i}$ and $v_{j}$ 
are also faraway, the value of $\left\|v_{i}-v_{j}\right\|^{2}$ must be as large as possible. Inspired by this observation, we propose a new distant repulsion assumption, which emphasizes that the lowdimensional counterpart of distant data pairs is also far apart in embedding subspace. This distant repulsion constraint is characterized by the objective function defined in

$$
\max _{V} \frac{1}{2} \sum_{i, j=1}^{N}\left\|v_{i}-v_{j}\right\|^{2} w_{i j}^{c} .
$$

The distant repulsion assumption in (9) can maintain the repellent relationship between each remote data pair during dimensionality reduction, which can be rewritten as

$$
\begin{aligned}
& \max _{V} \frac{1}{2} \sum_{i, j=1}^{N}\left\|v_{i}-v_{j}\right\|^{2} w_{i j}^{c} \\
& =\max _{V}\left(\sum_{i=1}^{N} v_{i}^{T} v_{i} d_{i i}^{c}-\sum_{i, j=1}^{N} v_{i}^{T} v_{j} w_{i j}^{c}\right) \\
& =\max _{V}\left(\operatorname{Tr}\left(V^{T} D^{C} V\right)-\operatorname{Tr}\left(V^{T} W^{C} V\right)\right) \\
& =\max _{V} \operatorname{Tr}\left(V^{T} L^{C} V\right),
\end{aligned}
$$

where $D^{C}$ and $L^{C}$ are the diagonal matrix and Laplacian matrix of graph $G^{C}$, respectively, and $L^{C}=D^{C}-W^{C}, d_{i i}^{C}=$ $\sum_{i \neq j} w_{i j}^{C}$ for any given $i$.

With the assumption that semantic label of distant data pairs is certainly different, (10) actually emphasizes the unfavorite relationship among dissimilar data samples.

3.2. Algorithm. Although (7) and (10) together can better preserve the manifold structure of data distribution, their optimized object is opposite, one for minimum and the other for maximum. To address this issue, we divide the basis matrix $U$ into two parts; that is,

$$
U=\left[U^{1}, U^{2}\right]
$$

correspondingly, the coefficient matrix $V$ is also partitioned into two parts:

$$
V=\left[V^{1}, V^{2}\right]
$$

Then, the original matrix $X$ can be reconstructed with $\left(U^{1}, V^{1}\right)$ and $\left(U^{2}, V^{2}\right)$ in an additive manner:

$$
X \approx\left[U^{1}, U^{2}\right]\left[V^{1}, V^{2}\right]^{T}=U^{1} V^{1^{T}}+U^{2} V^{2^{T}},
$$

where $U^{1} \in \mathbb{R}_{+}^{M \times Q}, V^{1} \in \mathbb{R}_{+}^{N \times Q}, U^{2} \in \mathbb{R}_{+}^{M \times(K-Q)}, V^{2} \in$ $\mathbb{R}_{+}^{N \times(K-Q)}$, and $K \geq Q . V^{1}$ and $V^{2}$ are the coefficient matrix of the original matrix $X$ on $U^{1}$ and $U^{2}$, respectively.

If we restrict the fact that the submatrix $V^{1}$ retains the property of graph $G$ and avoids the property of graph $G^{C}$, then submatrix $V^{1}$ is the low-dimensional representation of matrix $X$ with local invariance assumption. With the added constraint that the property of basic matrices $U^{1}$ and $U$ is consistent, (7) can be translated as

$$
\min _{V^{1}} \sum_{i, j=1}^{N}\left\|v_{i}^{1}-v_{j}^{1}\right\|^{2} W_{i j}=\min _{V^{1}} \operatorname{Tr}\left(V^{1^{T}} L V^{1}\right) .
$$

On the contrary, we assume that submatrix $V^{2}$ preserves the property of graph $G^{C}$ and avoids the property of graph $G$; meanwhile, we constrain the fact that the property of matrix $U^{2}$ is complementary to matrix $U$. This will lead to the fact that the calculation of finding maximum value in (11) is transformed into the calculation of finding minimum value showed in

$$
\min _{V^{2}} \sum_{i, j=1}^{N}\left\|v_{i}^{2}-v_{j}^{2}\right\|^{2} W_{i j}^{c}=\min _{V^{2}} \operatorname{Tr}\left(V^{2^{T}} L^{C} V^{2}\right) .
$$

In (2), the error of each data sample entered the objective function of NMF with squared residue, a few outliers with large errors will easily dominate the objective function. In this paper, we employ $\ell_{2,1}$-norm to define the data reconstruction term of NMF framework. Compared with $\ell_{2}$-norm, $\ell_{2,1}$ norm can keep the feature rotation invariance together with minimizing outlier impact. The $\ell_{2,1}$-norm of matrix $X$ can be defined as

$$
\|X\|_{2,1}=\sum_{i=1}^{N}\left\|x_{i}\right\|_{2}
$$

correspondingly, the $\ell_{2,1}$ robust loss function of NMF can be formulated as

$$
O=\left\|X-U V^{T}\right\|_{2,1}
$$

With the purpose of finding a robust low-dimensional representation with the nature of structure preservation, we explicitly incorporate (14) and (15) into the robust NMF framework. The objective function of the proposed RSPNMF is described as follows:

$$
\begin{aligned}
& O_{L} \\
= & \left\|X-U V^{T}\right\|_{2,1}^{2}+\alpha \operatorname{Tr}\left(V^{1^{T}} L V^{1}\right) \\
& +\beta \operatorname{Tr}\left(V^{2^{T}} L^{C} V^{2}\right) \\
\text { s.t. } \quad U & =\left[U^{1}, U^{2}\right] \geq 0, \\
V & =\left[V^{1}, V^{2}\right] \geq 0, \\
\alpha & \geq 0, \\
\beta & \geq 0 .
\end{aligned}
$$

From (18), we can find that RSPNMF divides the ultimate matrix factorization framework into two parts, one for structure preservation and the other for data reconstruction. The constants $\alpha$ and $\beta$ are the tradeoff parameters to balance the contribution of different regularization terms. When 
$\beta=0$, RSPNMF degenerates to GNMF [6]. When $\alpha=0$ and $\beta=0$, RSPNMF even degrade to the same form of NMF [23].

Because the objective function showed in (18) is not convex on both $U$ and $V$ together, in the following, we will manage to achieve its local minima with multiplicative iterative update algorithm.

Let $\psi_{i k}$ and $\phi_{j k}$ be the Lagrange multiplier for constraints $u_{i k} \geq 0$ and $v_{j k} \geq 0$, respectively, and $\Psi=\left[\psi_{i k}\right]$ and $\Phi=$ $\left[\phi_{j k}\right]$ are corresponding Lagrange multiplier matrix and the Lagrangian function $\mathscr{L}$ of $\mathrm{O}_{L}$ can be expressed as

$$
\mathscr{L}=O_{L}+\operatorname{Tr}\left(\Psi U^{T}\right)+\operatorname{Tr}\left(\Phi V^{T}\right)
$$

The partial derivatives of $\mathscr{L}$ with respect to $U$ and $V^{1}, V^{2}$ are as follows:

$$
\begin{aligned}
\frac{\partial \mathscr{L}}{\partial U}= & -2(\Lambda \odot X) V+2\left[\Lambda \odot\left(U V^{T}\right)\right] V+\psi, \\
\frac{\partial \mathscr{L}}{\partial V^{1}}= & -2(\Lambda \odot X)^{T} U^{1}+2\left[\Lambda \odot\left(U^{1} V^{1^{T}}\right)\right]^{T} U^{1} \\
& +2\left[\Lambda \odot\left(U^{2} V^{2^{T}}\right)\right]^{T} U^{1}+2 \alpha L V^{1}+\Phi, \\
\frac{\partial \mathscr{L}}{\partial V^{2}}= & -2(\Lambda \odot X)^{T} U^{2}+2\left[\Lambda \odot\left(U^{1} V^{1^{T}}\right)\right]^{T} U^{2} \\
& +2\left[\Lambda \odot\left(U^{2} V^{2^{T}}\right)\right]^{T} U^{2}+2 \beta L^{C} V^{2}+\Phi,
\end{aligned}
$$

where $\Lambda=\left[\left(1 /\left\|\left(X-U V^{T}\right){ }_{.1}\right\|\right) I_{M \times 1}, \ldots,(1 / \|(X-\right.$ $\left.\left.\left.U V^{T}\right)_{\cdot N} \|\right) I_{M \times 1}\right]$ and $I_{M \times 1}$ is a column vector with all elements equal to one.

Using the KKT conditions $\psi_{i k} u_{i k}=0, \phi_{j k} v_{j k}^{1}=0$, and $\phi_{j k} v_{j k}^{2}=0$, we obtain the following multiplicative update rules for RSPNMF:

$$
\begin{aligned}
& u_{i k} \longleftarrow u_{i k} \frac{[(\Lambda \odot X) V]_{i k}}{\left[\left(\Lambda \odot\left(U V^{T}\right)\right) V\right]_{i k}}, \\
& v_{j k} \longleftarrow v_{j k} \frac{\left[(\Lambda \odot X)^{T} U+\left(\alpha W V^{1} \beta W^{C} V^{2}\right)\right]_{j k}}{\left[\left(\Lambda \odot\left(U V^{T}\right)\right)^{T} U+\left(\alpha D V^{1} \beta D^{C} V^{2}\right)\right]_{j k}} .
\end{aligned}
$$

To avoid the scale transfer problem which was pointed out in [44], we normalize $U$ and $V$ as follows:

$$
\begin{aligned}
& V_{i \cdot}^{t+1} \longleftarrow V_{i \cdot}^{t+1} \times\left\|U_{\cdot i}^{t+1}\right\|_{2}, \\
& U_{\cdot i}^{t+1} \longleftarrow \frac{U_{\cdot i}^{t+1}}{\left\|U_{\cdot i}^{t+1}\right\|_{2}} .
\end{aligned}
$$

The total procedure for solving the minimization problem of (18) is presented in Algorithm 1, from which we can obtain a low-dimensional representation of input data samples.

3.3. Convergence Analysis. Due to the objective function shown in (18) is nonconvex, only local optimal solutions can be obtained. In this section, we use the property of auxiliary function approach to prove the objective function $O_{L}$ is nonincreasing under the update formulas in (21) and (22).

\section{Convergence Analysis for $U$}

Lemma 1. If there exists an auxiliary function $G\left(u, u^{t}\right)$ for $F(u)$ which satisfies $G\left(u, u^{t}\right) \geq F(u)$ and $G(u, u)=F(u)$, then $F(u)$ is nonincreasing under the update

$$
u^{t+1}=\underset{u}{\arg \min } G\left(u, u^{t}\right)
$$

Proof. Consider $F\left(u^{t+1}\right) \leq G\left(u^{t+1}, u^{t}\right) \leq G\left(u^{t}, u^{t}\right)=F\left(u^{t}\right)$.

In order to prove the objective function $O_{L}$ is nonincreasing under the update formula in (21), we only need to construct a proper auxiliary function $G_{a b}\left(U, U^{t}\right)$ which satisfies $G_{a b}\left(U, U^{t}\right) \geq F_{a b}(U)$ and $G_{a b}(U, U)=F_{a b}(U)$, where $F(U)$ denotes the part of $O_{L}$ which is only relevant to $U$ and $F_{a b}(U)$ is the $(a, b)$ th element of $F(U)$. as

The Taylor series expansion of $F_{a b}(U)$ can be formulated

$$
\begin{aligned}
F_{a b}(U)= & F_{a b}\left(U^{t}\right)+F_{a b}^{\prime}\left(U^{t}\right)\left(u_{a b}-u_{a b}^{t}\right) \\
& +\frac{1}{2} F_{a b}^{\prime \prime}\left(U^{t}\right)\left(u_{a b}-u_{a b}^{t}\right)^{2}
\end{aligned}
$$

where

$$
\begin{aligned}
F_{a b}^{\prime}\left(U^{t}\right) & =\left(\frac{\partial O_{L}}{\partial U}\right)_{a b} \\
& =\left\{2\left[\Lambda \odot\left(U V^{T}\right)\right] V-2[\Lambda \odot X] V\right\}_{a b}, \\
F_{a b}^{\prime \prime}\left(U^{t}\right) & =\left(\frac{\partial F_{a b}^{\prime}\left(U^{t}\right)}{\partial U}\right)_{a b}=[\Lambda(V \odot V)]_{a b} .
\end{aligned}
$$

Lemma 2. Function

$$
\begin{aligned}
G_{a b}\left(U, U^{t}\right)= & F_{a b}\left(U^{t}\right)+F_{a b}^{\prime}\left(U^{t}\right)\left(u_{a b}-u_{a b}^{t}\right) \\
& +\frac{\left\{\left[\Lambda \odot\left(U V^{T}\right)\right] V\right\}_{a b}}{u_{a b}^{t}}\left(u_{a b}-u_{a b}^{t}\right)^{2}
\end{aligned}
$$

is an auxiliary function for $F_{a b}(U)$ which satisfies $G_{a b}\left(U, U^{t}\right) \geq$ $F_{a b}(U)$ and $G_{a b}\left(U, U^{t}\right)=F_{a b}(U)$.

Proof. Obviously, $G_{a b}(U, U)=F_{a b}(U)$ is true; we only need to prove $G_{a b}\left(U, U^{t}\right) \geq F_{a b}(U)$. For this purpose, we compare $G_{a b}\left(U, U^{t}\right)$ with the Taylor series expansion of $F_{a b}(U)$.

Since

$$
\begin{aligned}
& {[\Lambda(V \odot V)]_{a b} u_{a b}^{t}=\left(\sum_{j} \Lambda_{a j} v_{j b} v_{j b}\right) u_{a b}^{t},} \\
& \left\{\left[\Lambda \odot\left(U V^{T}\right)\right] V\right\}_{a b}=\sum_{j} \Lambda_{a j} v_{j b}\left(\sum_{k} u_{a k} v_{j k}\right) \\
& \quad=\sum_{j} \Lambda_{a j} v_{j b}\left(u_{a b}^{t} v_{j b}+\left(\sum_{k \neq b} u_{a k} v_{j k}\right)\right),
\end{aligned}
$$


(1) Initialize $U^{0}, V^{0}$ as arbitrary non-negative matrices.

(2) For $t=0,1,2, \ldots, T$, Do

(1) For given $V=V^{t}$, update the basis matrix $U^{t+1}$ according to (21).

(2) For given $U=U^{t}$, update the basis matrix $V^{t+1}$ according to (22).

(3) Normalize $U^{t+1}$ and $V^{t+1}$ according to (23).

(3) Output $U=U^{T}$ and $V=V^{T}$.

Algorithm 1: RSPNMF algorithm description.

we can find that $\left\{[\Lambda(V \odot V)]_{a b}\right\}_{a b} u_{a b}^{t} \leq\left\{\left[\Lambda \odot\left(U V^{T}\right)\right] V\right\}_{a b}$, and then we can conclude that $G_{a b}\left(U, U^{t}\right) \geq F_{a b}(U)$.

From Lemma 2, we can find that $F_{a b}(U)$ is nonincreasing under the update rule in (21).

Let $\partial G_{a b}\left(U, U^{t}\right) / \partial u_{a b}=0$; there must be $u^{*}$, which makes $G_{a b}\left(U, U^{t}\right)$ attain the minima, so we can obtain that

$$
\begin{aligned}
u^{*} & =u_{a b}^{t}-u_{a b}^{t} \frac{F_{a b}^{\prime}\left(u_{a b}^{t}\right)}{2\left\{\left[\Lambda \odot\left(U V^{T}\right)\right] V\right\}_{a b}} \\
& =u_{a b}^{t}-u_{a b}^{t} \frac{\left\{2\left[\Lambda \odot\left(U V^{T}\right)\right] V-2[\Lambda \odot X] V\right\}_{a b}}{2\left\{\left[\Lambda \odot\left(U V^{T}\right)\right] V\right\}_{a b}} \\
& =u_{a b} \frac{[(\Lambda \odot X) V]_{a b}}{\left[\left(\Lambda \odot U V^{T}\right) V\right]_{a b}} .
\end{aligned}
$$

Convergence Analysis for $V^{1}$. Similar to the convergence analysis for $U$, the first and the second derivate of $O_{L}$ with respect to $V^{1}$ can be described as

$$
\begin{aligned}
& F_{a b}^{\prime}\left(V^{1}\right)=\left(\frac{\partial O_{L}}{\partial V^{1}}\right)_{a b}=\left\{-2(\Lambda \odot X)^{T} U^{1}\right. \\
& \left.+2\left[\Lambda \odot\left(U V^{T}\right)\right]^{T} U^{1}+2(D-W) V^{1}\right\}_{a b}, \\
& F_{a b}^{\prime \prime}\left(V^{1}\right)=\left(\frac{\partial F_{a b}^{\prime}\left(V^{1}\right)}{\partial V^{1}}\right)_{a b}=\left[\Lambda^{T}\left(U^{1} \odot U^{1}\right)\right]_{a b} \\
& \quad+2(D-W)_{a a} .
\end{aligned}
$$

The Taylor series expansion of $F_{a b}\left(V^{1}\right)$ is as follows:

$$
\begin{aligned}
F_{a b}\left(V^{1}\right)= & F_{a b}\left(V^{1^{t}}\right)+F_{a b}^{\prime}\left(V^{1^{t}}\right)\left(v_{a b}^{1}-v_{a b}^{1^{t}}\right) \\
& +\frac{1}{2} F_{a b}^{\prime \prime}\left(V^{1^{t}}\right)\left(v_{a b}^{1}-v_{a b}^{1^{t}}\right)^{2} .
\end{aligned}
$$

Now, we construct a new auxiliary function for $F_{a b}\left(V^{1}\right)$ :

$$
\begin{aligned}
& G_{a b}\left(V^{1}, V^{1^{t}}\right) \\
& =F_{a b}\left(V^{1^{t}}\right)+F_{a b}^{\prime}\left(V^{1^{t}}\right)\left(v_{a b}^{1}-v_{a b}^{1^{t}}\right) \\
& +\frac{\left\{\left[\Lambda \odot\left(U V^{T}\right)\right]^{T} U_{1}+\alpha D V^{1}\right\}_{a b}}{v_{a b}^{1^{t}}}\left(v_{a b}^{1}-v_{a b}^{1^{t}}\right)^{2},
\end{aligned}
$$

and we prove which satisfies $G_{a b}\left(V^{1}, V^{1^{t}}\right) \geq F_{a b}\left(V^{1}\right)$ and $G_{a b}\left(V^{1}, V^{1}\right) \geq F_{a b}\left(V^{1}\right)$.

Proof. Obviously, $G_{a b}\left(V^{1}, V^{1}\right)=F_{a b}\left(V^{1}\right)$ is true; we only need to prove $G_{a b}\left(V^{1}, V^{1^{t}}\right) \geq F_{a b}\left(V^{1}\right)$.

Since

$$
\begin{aligned}
{\left[\Lambda^{T}\left(U^{1} \odot U^{1}\right)\right]_{a b} v_{a b}^{1^{t}} } & \leq\left\{\left[\Lambda \odot\left(U V^{T}\right)\right]^{T} U^{1}\right\}_{a b}, \\
(D-W)_{a a} v_{a b}^{1^{t}} & \leq\left(D V^{1}\right)_{a b},
\end{aligned}
$$

we can deduce that $G\left(v_{a b}^{1}, v_{a b}^{1^{t}}\right) \geq F_{a b}\left(v_{a b}^{1}\right)$.

Let $\partial G_{a b}\left(V^{1}, V^{1^{t}}\right) / \partial v_{a b}^{1}=0$; we can find that

$$
\begin{aligned}
& F_{a b}\left(V^{1^{t}}\right) \\
& \quad+2 \frac{\left\{\left[\Lambda \odot\left(U V^{T}\right)\right]^{T} U_{1}+\alpha D V_{a b}^{1}\right\}_{a b}}{v_{a b}^{1^{t}}}\left(v_{a b}^{1}-v_{a b}^{1^{t}}\right) \\
& \quad=0 .
\end{aligned}
$$

Finally, we have

$$
v_{a b}^{1} \longleftarrow v_{a b}^{1} \frac{\left[(\Lambda \odot X)^{T} U^{1}+\alpha W V^{1}\right]_{a b}}{\left[\left(\Lambda \odot U V^{T}\right)^{T} U^{1}+\alpha D V^{1}\right]_{a b}} .
$$

The convergence analysis for $V^{2}$ is similar to the convergence analysis for $V^{1}$, and it is omitted.

Computational Complexity Analysis. With the update rules in (21) and (22), it is not hard to calculate the extra computational cost of our proposed algorithm in each iteration, and we summarize the result in Table 1. It is important to note that matrix $W$ is sparse, because the local graph $G$ is constructed with $k_{1}$ neighbors. The average nonzero element on each row of $W$ is $k_{1}$. Thus, we only need $N k_{1} Q$ and flam (a floating point addition and multiplication) to compute $W V^{1}$.

Besides the multiplicative updates, RSPNMF also needs $O\left(M N^{2}\right)$ to construct the local affinity graph $G$ and distant repulsion graph $G^{C}$. If the multiplicative updates stop after $t$ iterations, the overall cost for RSPNMF is $O\left(t M N K+2 M N^{2}\right)$. The overall cost for GNMF is $O\left(t M N K+M N^{2}\right)$ and the overall cost for SPNMF is $O\left(t(M+N) N K+M N^{2}\right)$. 
TABLE 1: Computational operation counts for updating $U$ and $V$ in each iteration.

\begin{tabular}{|c|c|c|c|c|}
\hline & Addition & Multiplication & Division & Overall \\
\hline Updating $U(21)$ & $3 M N K-M(N+2 K)$ & $3 M N K+2 M N$ & $M K$ & $O(M N K)$ \\
\hline Updating $V(22)$ & $\begin{array}{l}2 N K(M-1)+M N(K-1) \\
+N Q\left(k_{1}-1\right)+N^{2}(K-Q)\end{array}$ & $\begin{array}{c}3 M N K+N Q\left(k_{1}+1\right) \\
+N^{2}(K-Q)\end{array}$ & $N K$ & $O(M N K)$ \\
\hline Normalizing $U$ and $V$ & $(M-1) N$ & $M N$ & $M K$ & $O(M N)$ \\
\hline Overall & $O(M N K)$ & $O(M N K)$ & $O((M+N) K)$ & \\
\hline
\end{tabular}

Addition: a floating-point addition; multiplication: a floating-point multiplication; division: a floating-point division.

TABLE 2: Statistic of real-world datasets.

\begin{tabular}{lccc}
\hline Datasets & \# samples & \# features & \# class \\
\hline ORL & 400 & 1024 & 40 \\
YALE & 165 & 1024 & 15 \\
FERET & 420 & 1024 & 70 \\
\hline
\end{tabular}

\section{Experimental Results}

In this section, we carry out several experiments to examine the clustering performance of our approach in comparison with some state-of-the-art methods.

4.1. Datasets Description. Three image datasets which are widely employed in dimensionality reduction literatures are used in our experiments.

(1) ORL Dataset. The ORL dataset contains 400 face images of 40 subjects under different lighting, facial expressions (open/closed eyes, smiling/not smiling), and facial details (with glasses or no glasses). All these images were taken against a dark homogeneous background with the subjects in an upright, frontal position. In our experiments, original images are firstly normalized in scale and orientation, such that two eyes are aligned at the same position. Then, the facial areas are cropped into the final images for clustering. Finally, each image is normalized to array of $32 \times 32$ pixels and reshaped to a long vector with 256 gray levels per pixel.

(2) YALE Dataset. The YALE dataset includes 165 face images collected from 15 volunteers. Each image has different facial expression and facial details. The same preprocessing as for the ORL dataset is implemented on YALE dataset.

(3) FERET Dataset. For the FERET dataset, we use 70 subjects with 6 facial images for each subject. Each image is also represented by a 1024-dimensional vector in image space.

The statistic property of these datasets is listed in Table 2, which includes the number of samples, the dimension of features, and the number of object classes.

As showed in Figure 1, to evaluate the robustness of mentioned algorithms to noisy data, all the images were occluded with local and global noise. The local noise consists of occlusion patch of $12 \times 12$ pixels with random black and white dots. The global noise is replaced by Gaussian noise with mean 0 and variance 36 .
4.2. Compared Methods. To investigate the clustering performance of our proposed approach, we compared it with the following methods:

(i) Canonical $K$-means (Kmeans in short) clustering method as a baseline.

(ii) $K$-means clustering in the principle component subspace (PCA in short).

(iii) Nonnegative matrix factorization based clustering (NMF in short). We use the $F$-norm formulation.

(iv) Graph regularized nonnegative matrix factorization based clustering with $F$-norm formulation (GNMF in short).

(v) Structure preserving nonnegative matrix factorization based clustering (SPNMF in short).

Except the Kmeans, for each image, a new lowdimensional representation can be derived from the matrix factorization, and then we perform clustering by using $K$ means in the new embedding subspace.

4.3. Evaluation Metrics. To evaluate the clustering performance of all involved methods, we adopted two popular metrics, the accuracy (AC) and the normalized mutual information (NMI), as used in [6].

The accuracy indicates one-to-one relationship between obtained cluster label and ground truth label. The accuracy is defined as follows:

$$
\mathrm{AC}=\frac{\sum_{i=1}^{n} \delta\left(\operatorname{map}\left(r_{i}\right), l_{i}\right)}{N},
$$

where $\delta\left(\operatorname{map}\left(r_{i}\right), l_{i}\right)$ is the delta function which judges whether the ground truth $l_{i}$ is equal to the obtained label $r_{i}$ and $\operatorname{map}\left(r_{i}\right)$ is a permutation mapping function that maps each obtained cluster label $r_{i}$ to the equivalent label from the dataset.

The second measure metrics is the normalized mutual information (NMI), which is used for determining the quality of clustering.

Let $C$ and $C^{\prime}$ denote the sets of cluster from the ground truth and the clustering method, respectively. The mutual information metric $\mathrm{MI}\left(C, C^{\prime}\right)$ is defined as follows:

$$
\operatorname{MI}\left(C, C^{\prime}\right)=\sum_{c_{i} \in C, c_{j}^{\prime} \in C^{\prime}} p\left(c_{j}, c_{j}^{\prime}\right) \cdot \log _{2} \frac{p\left(c_{j}, c_{j}^{\prime}\right)}{p\left(c_{j}\right) \cdot p\left(c_{j}^{\prime}\right)},
$$




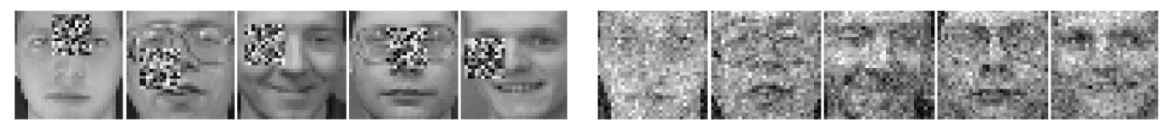

(a)

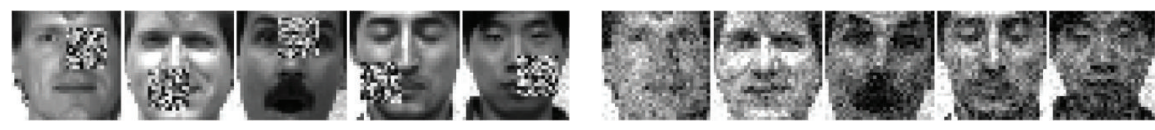

(b)
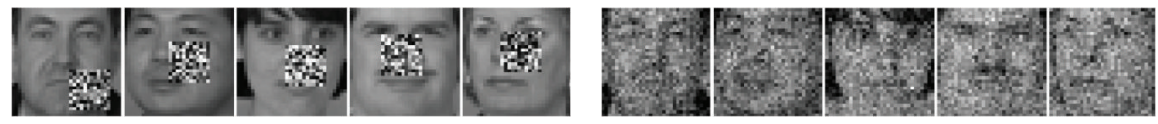

(c)

Figure 1: Sample images from ORL (a), YALE (b), and FERET (c).

where $p\left(c_{j}\right)$ and $p\left(c_{j}^{\prime}\right)$ denote the extent of a sample belonging to the clusters $c_{i}$ and $c_{j}^{\prime}$, respectively. $p\left(c_{j}, c_{j}^{\prime}\right)$ is a joint probability that an arbitrarily selected sample belongs to $c_{i}$ as well as $c_{j}^{\prime}$ at the same time. The NMI can be defined as follows:

$$
\operatorname{NMI}\left(C, C^{\prime}\right)=\frac{\operatorname{MI}\left(C, C^{\prime}\right)}{\max \left(H(C), H\left(C^{\prime}\right)\right)}
$$

where $H(C)$ and $H\left(C^{\prime}\right)$ are the entropies of $C$ and $C^{\prime}$, respectively. It can be seen that $\operatorname{NMI}\left(C, C^{\prime}\right)$ ranges from 0 to 1. The larger the NMI is, the better the clustering result will be.

4.4. Clustering Results and Analysis. In order to fair examine the clustering performance of all mentioned methods, 20 test runs are conducted on the optimal parameter setting, and then the means and standard deviations are recorded. In each test, Kmeans is applied 20 times with different initialization and the best result is reported. All clustering performances on the three noise contaminated datasets are reported in Tables 3 and 4 .

From these results, we can derive the following observations: (a) SPNMF outperforms Kmeans, PCA, and NMF in most cases. This demonstrates the importance of structure preservation in discovering the hidden factors. In addition, the performance of SPNMF is superior to GNMF. This reveals that, by leveraging the ratio of local affinity and distant repulsion regularization, SPNMF can learn a better low-dimensional representation. (b) The performance of RSPNMF exceeds SPNMF; this demonstrates the robustness of $\ell_{2,1}$-norm in handling noise data samples.

4.5. Parameter Sensitiveness. Our RSPNMF has five essential parameters: the number of nearest neighbors $k_{1}$, the number of distant neighbors $k_{2}$, the regularization parameter $\alpha$, the regularization parameter $\beta$, and the number of basis vectors $r$. For all mentioned dimensionality reduction algorithms, the number of basis vectors $r$ is equal to the number of clusters. The complement space and the original space reduced dimension $d$ is set to $r / 2$. For all graph-based methods, the number of nearest neighbors is set to $\{3,5, \ldots, 19,21\}$. For SPNMF and RSPNMF, the number of distant points is also set to $\{3,5, \ldots, 19,21\}$. For GNMF, the regularization parameter is set by the grid $\left\{10^{-3}, 10^{-2}, \ldots, 10^{3}\right\}$. For SPNMF and RSPNMF, we tune $\alpha$ and $\beta$ by searching the grid $\left\{10^{-3}, 10^{-2}, \ldots, 10^{3}\right\}$. For SPNMF, $\gamma$ is set to 1 .

The result of parameter sensitiveness on different datasets with local and global noise is showed in Figure 2. In order to compare with GNMF, we fix the parameter $\beta$ and tune the parameter $\alpha$. From Figure 2, we can find that the performance of RSPNMF and SPNMF is superior to GNMF; meanwhile, they are all stable with respect to the change of parameter $\alpha$. This demonstrates the importance of the distant repulsion preservation on characterizing the intrinsic distribution of data samples. Besides, RSPNMF still outperforms SPNMF when $\alpha$ range within $\{0.001,10\}$. This is because the robust data reconstruction term of RSPNMF alleviates the noisy data impact on clustering performance.

The relevant samples selection plays an important role in all graph regularized NMF method. For GNMF, which only use $k_{1}$-nearest neighboring graph to capture the local geometric structure of data distribution; meanwhile, assume that the neighboring samples are with the same semantic label. For noise corrupted data samples, this assumption may not hold. In particular, as $k_{1}$ increases, clustering performance of GNMF is more likely to fail as shown in Figure 3. For RSPNMF and SPNMF, the local and distant data samples relationship is also considered. This will definitely reinforce the algorithm robustness to $k_{1}$-nearest neighboring samples increase. In addition, from Figure 3 we can find that the introduction of $\ell_{2,1}$-norm improves RSPNMF clustering results.

4.6. Convergence and Computational Cost. The effectiveness of the proposed RSPNMF has been demonstrated by the above clustering experiments. Here, we evaluate the efficiency of RSPNMF, that is, how fast the update rules in (21) and (22) are converged.

Figure 4 shows the convergence curves of RSPNMF on all the three datasets. For each figure, the $y$-axis is the value of objective function and the $x$-axis denotes the iteration 

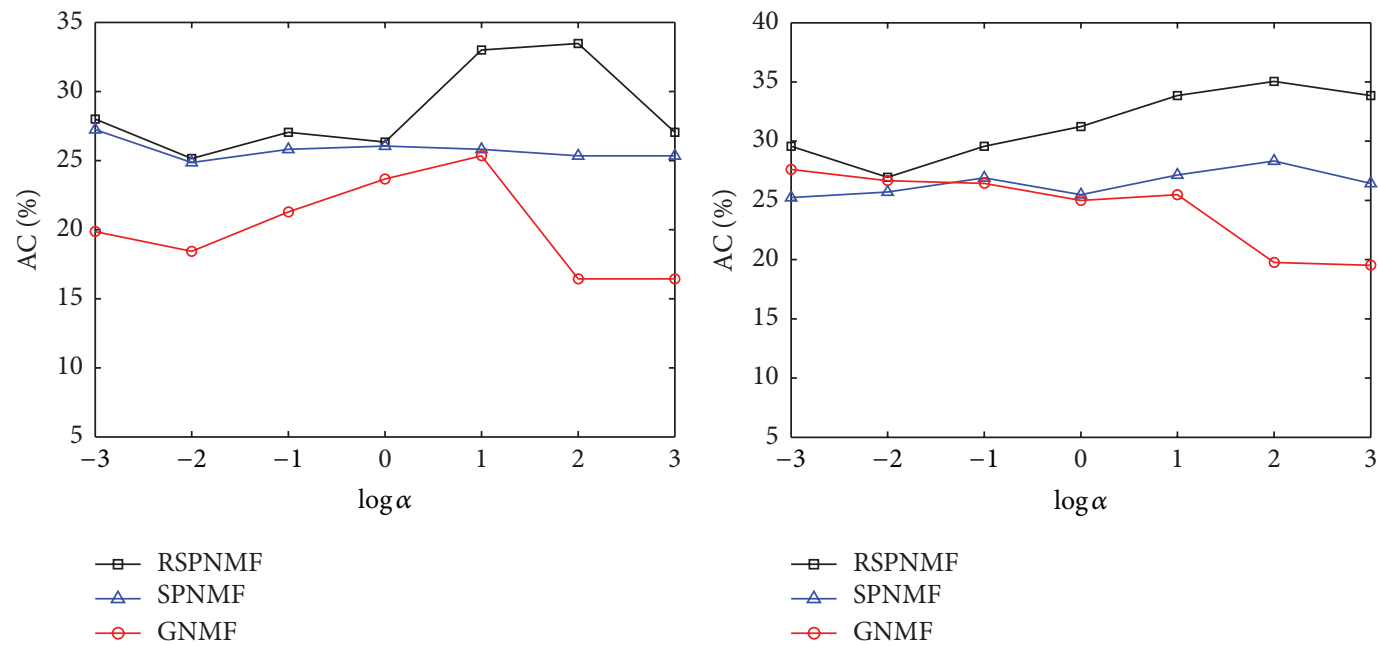

(a) FERET
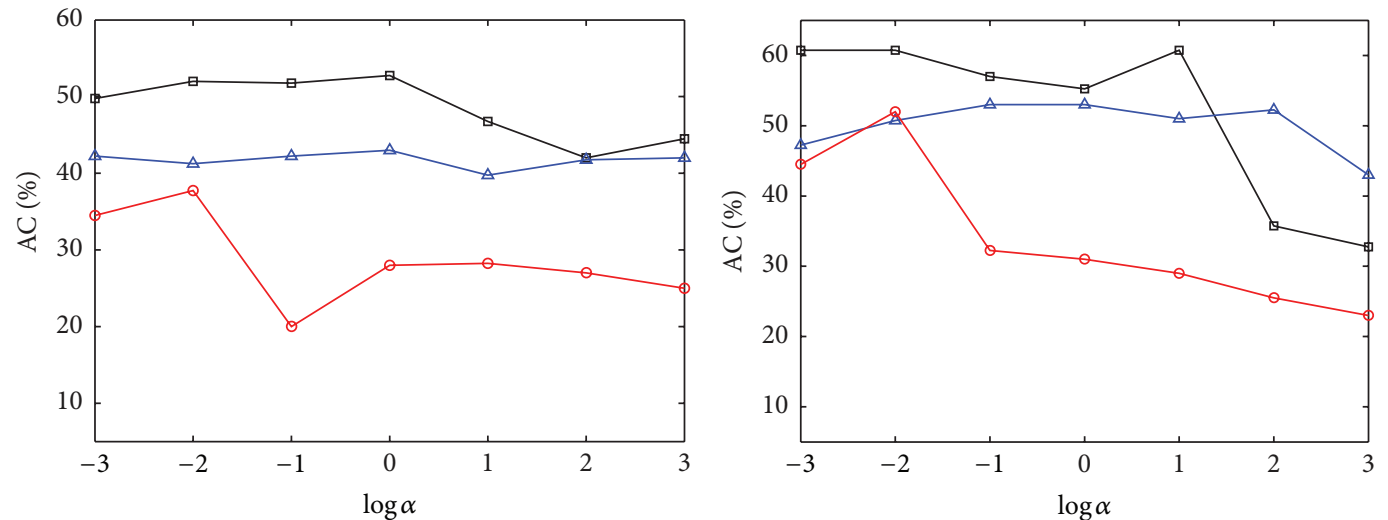

$$
\begin{aligned}
& \square \text { RSPNMF } \\
& \triangle \text { SPNMF } \\
& - \text { GNMF }
\end{aligned}
$$

$$
\begin{aligned}
& \square \text { RSPNMF } \\
& \triangle \text { SPNMF } \\
& - \text { GNMF }
\end{aligned}
$$

(b) ORL
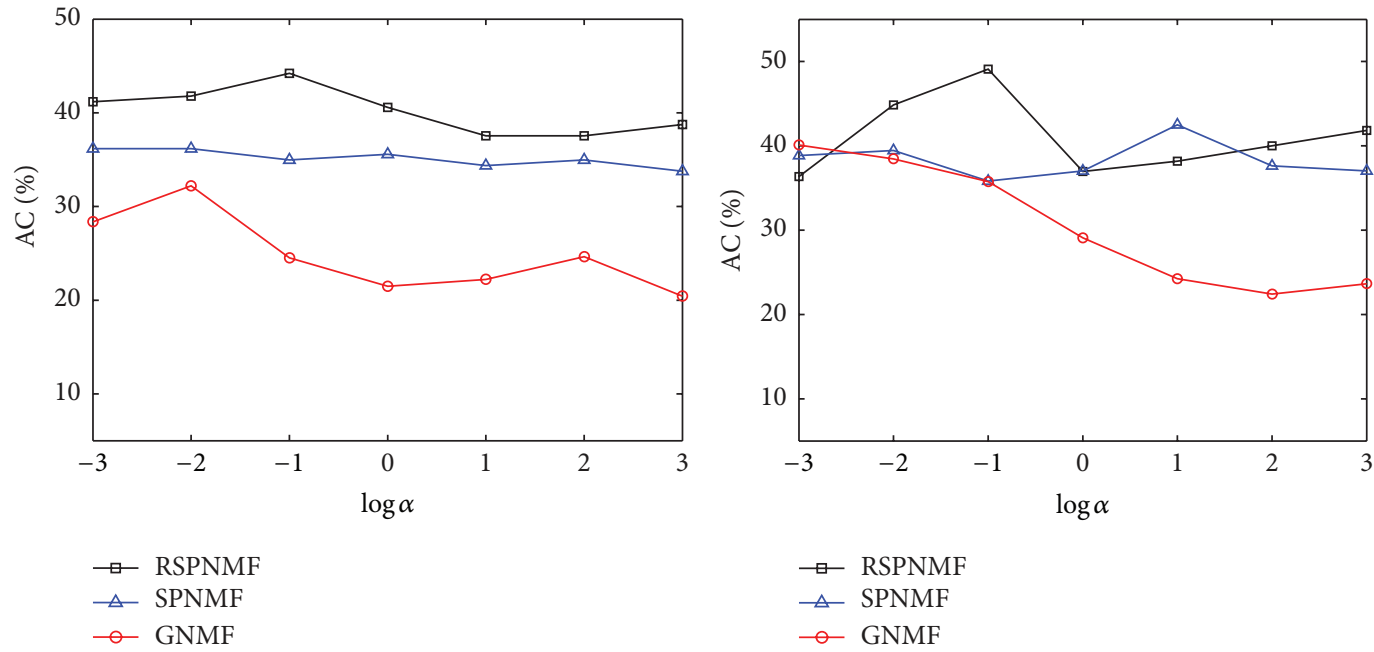

(c) YALE

FIGURE 2: The performance of involved methods with respect to the parameter $\alpha$ on different dataset. The result of local noise is shown in the first column. The result of global noise is shown in the second column. 

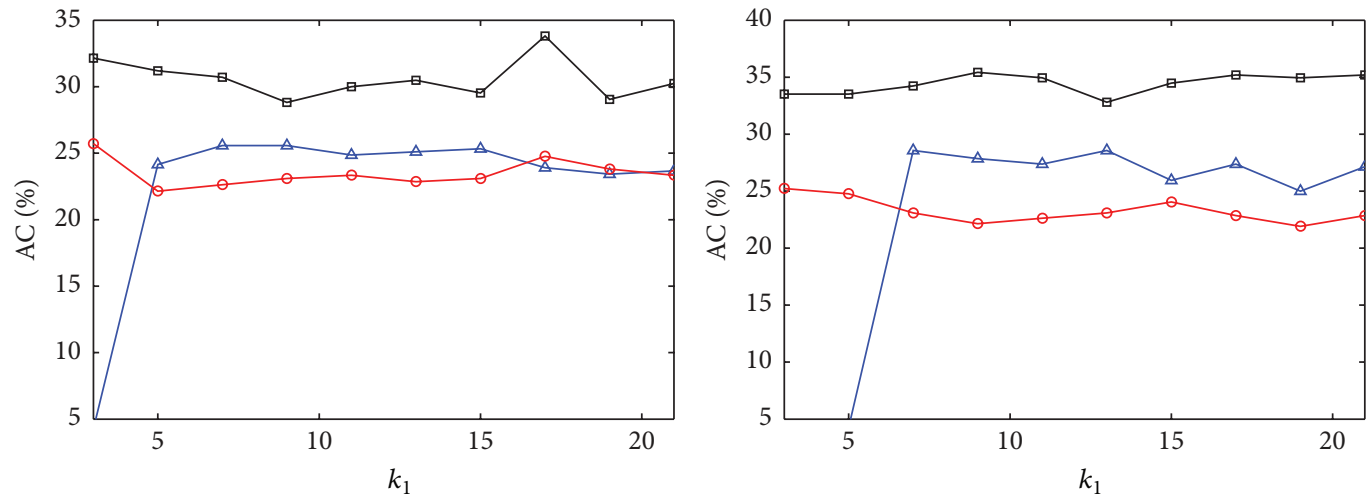

$$
\begin{aligned}
& \square \text { RSPNMF } \\
& \triangle \text { SPNMF } \\
& - \text { GNMF }
\end{aligned}
$$

$$
\begin{aligned}
& \square \text { RSPNMF } \\
& \triangle \text { SPNMF } \\
& - \text { GNMF }
\end{aligned}
$$

(a) FERET
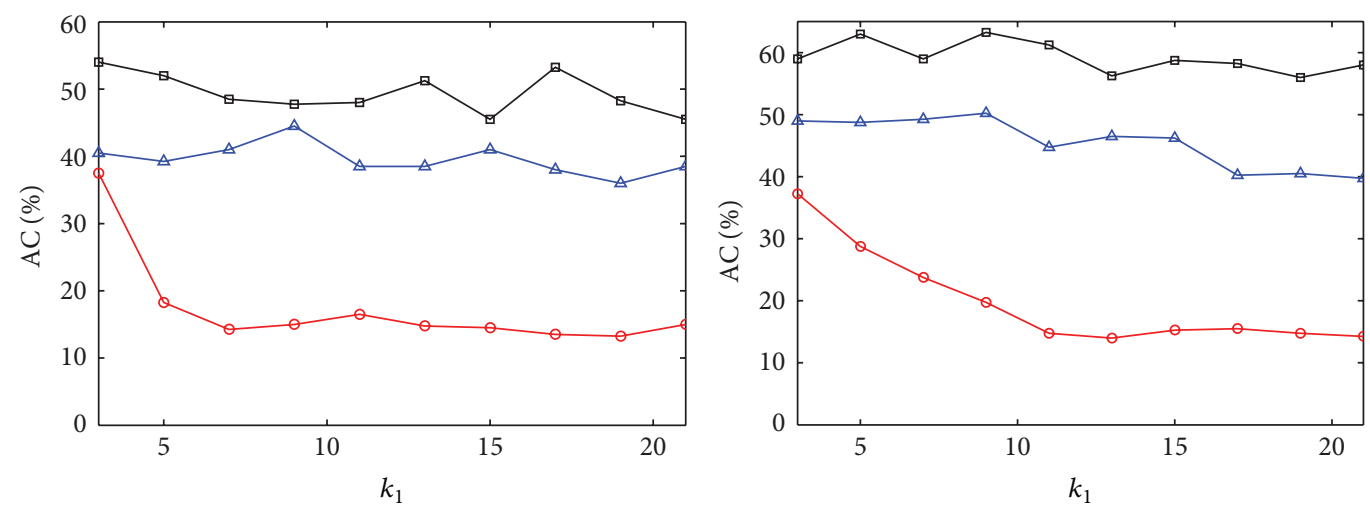

$$
\begin{aligned}
& \square \text { RSPNMF } \\
& \triangle \text { SPNMF } \\
& - \text { GNMF }
\end{aligned}
$$

$$
\begin{aligned}
& \square \text { RSPNMF } \\
& \triangle \text { SPNMF }
\end{aligned}
$$$$
- \text { GNMF }
$$

(b) ORL
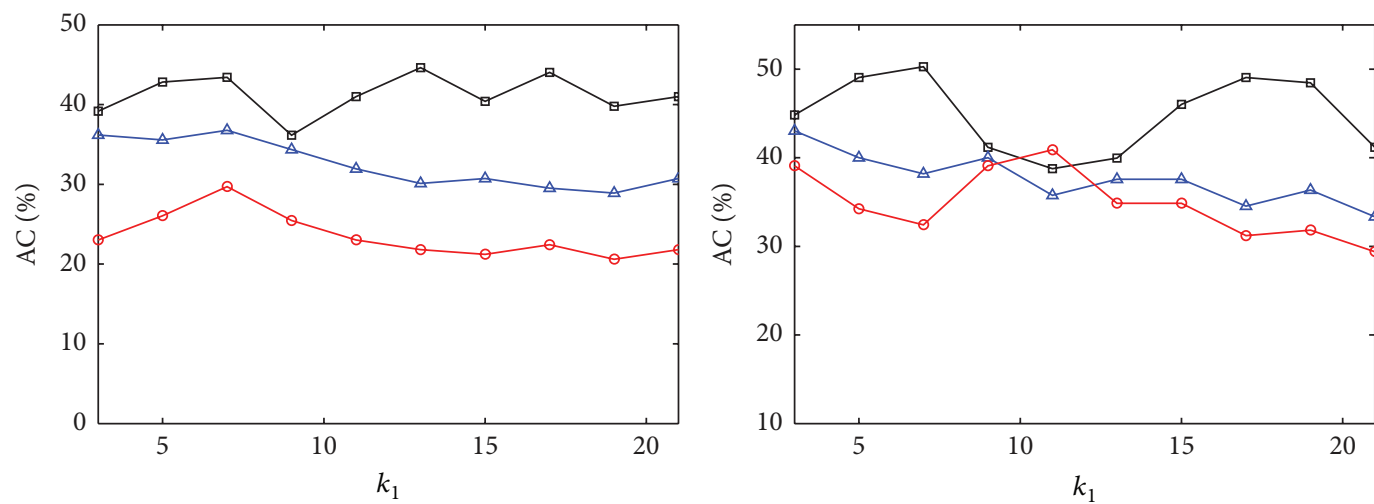

$$
\begin{aligned}
& \square \text { RSPNMF } \\
& \triangle \text { SPNMF } \\
& - \text { GNMF }
\end{aligned}
$$

$$
\begin{aligned}
& \square \text { RSPNMF } \\
& \triangle \text { SPNMF } \\
& - \text { GNMF }
\end{aligned}
$$

(c) YALE

Figure 3: The performance of involved methods with respect to parameter $k_{1}$. The result of local noise is shown in the first column. The result of global noise is shown in the second column. 


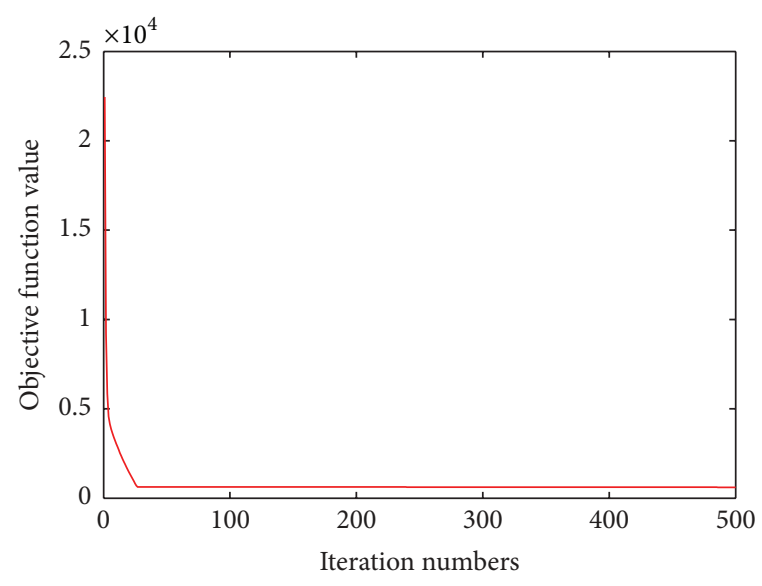

— RSPNMF

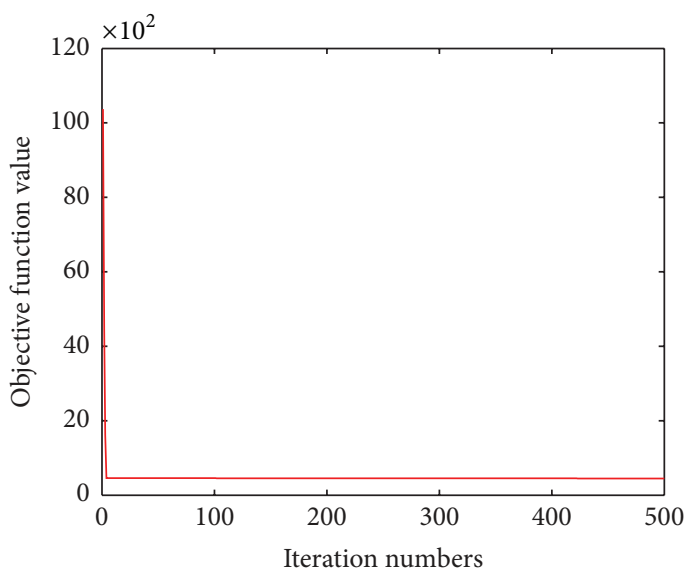

- RSPNMF

(a) FERET
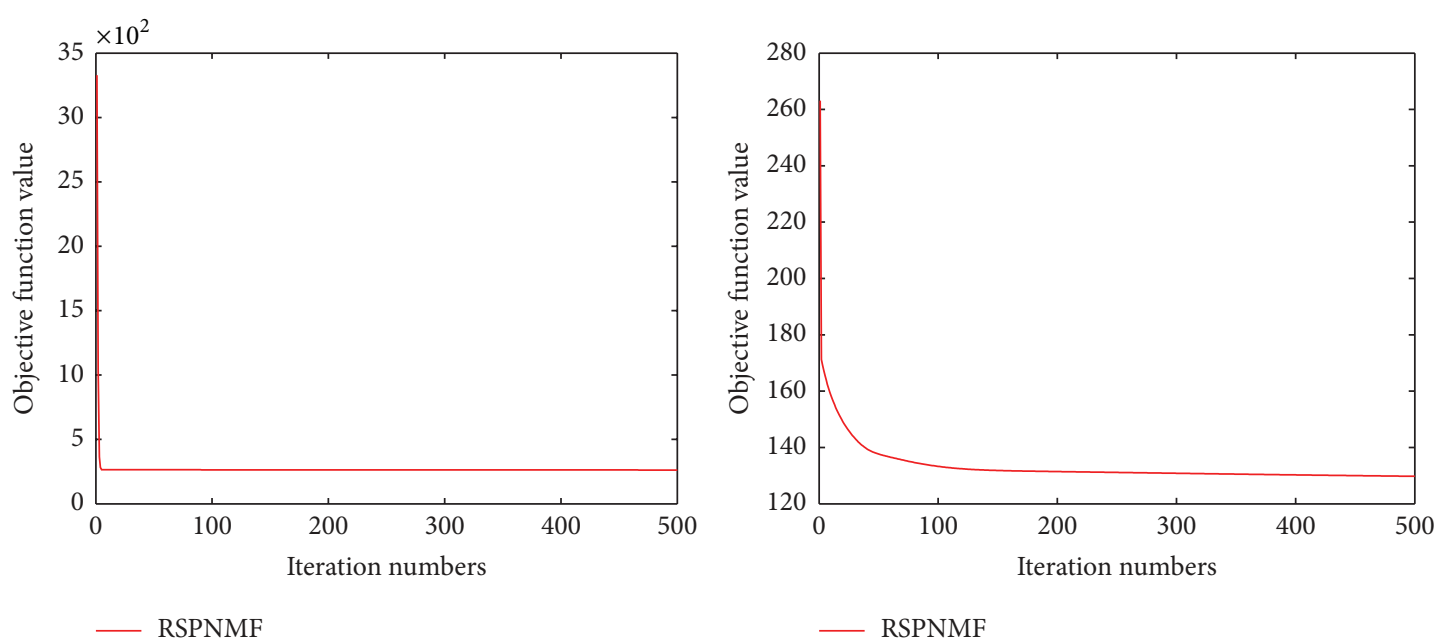

(b) ORL
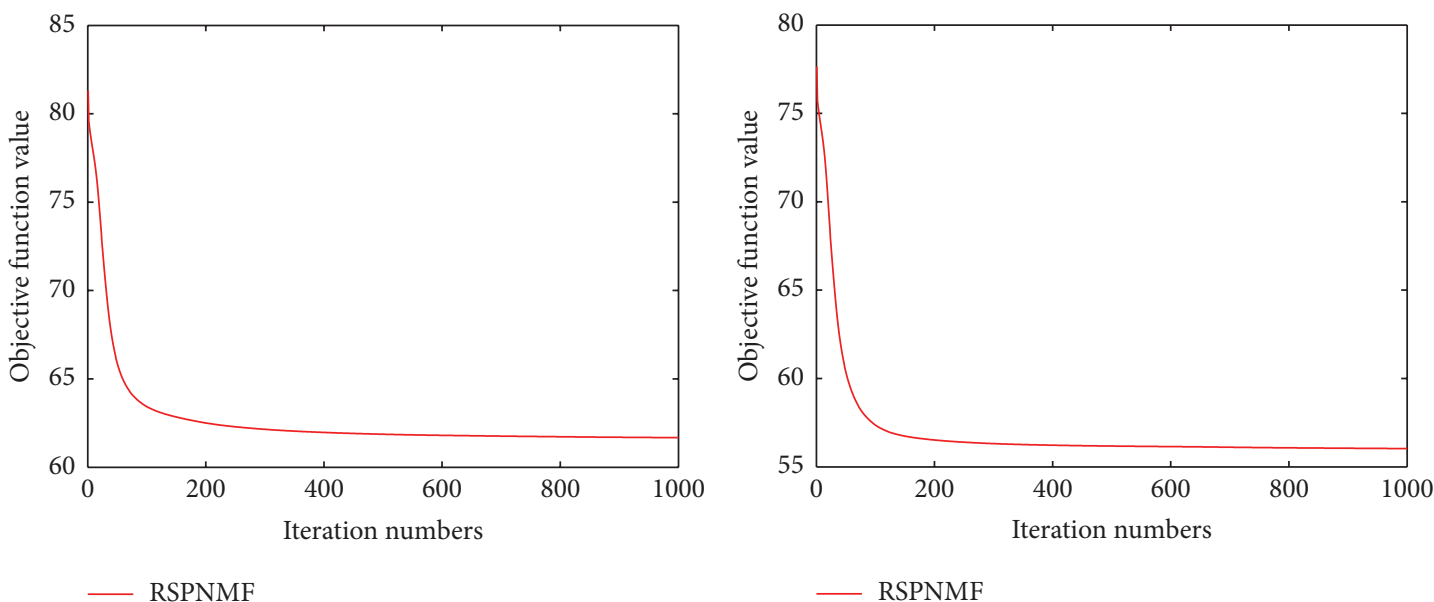

(c) YALE

FIgURE 4: The convergence curve of RSPNMF with 500 iterations. The result of local noise is shown in the first column. The result of global noise is shown in the second column. 
TABLE 3: Clustering performance on local noise contaminated datasets. The best performances are highlighted in bold font.

(a)

\begin{tabular}{lcccccc}
\hline \multirow{2}{*}{ Dataset } & & \multicolumn{2}{c}{ Accuracy (\%) } & & \\
& Kmeans & PCA & NMF & GNMF & SPNMF & RSPNMF \\
\hline FERET & $23.10 \pm 1.14$ & $26.12 \pm 1.27$ & $24.20 \pm 1.03$ & $25.05 \pm 0.74$ & $27.45 \pm 5.22$ & $\mathbf{3 3 . 0 6} \pm \mathbf{1 . 2 5}$ \\
ORL & $35.71 \pm 2.74$ & $46.99 \pm 2.69$ & $44.56 \pm 1.59$ & $37.78 \pm 1.37$ & $44.78 \pm 1.7$ & $\mathbf{5 4 . 0 1} \pm \mathbf{3 . 3}$ \\
Yale & $32.12 \pm 2.38$ & $37.43 \pm 2.27$ & $38.18 \pm 2.18$ & $32.45 \pm 1.16$ & $36.36 \pm 1.24$ & $\mathbf{4 4 . 4 2} \pm \mathbf{1 . 0 8}$ \\
\hline
\end{tabular}

(b)

\begin{tabular}{lccccrr}
\hline \multirow{2}{*}{ Dataset } & & \multicolumn{5}{c}{ Normalized mutual information (\%) } \\
& Kmeans & PCA & NMF & GNMF & SPNMF & RSPNMF \\
\hline FERET & $49.2 \pm 2.56$ & $56.85 \pm 1.54$ & $54.33 \pm 1.12$ & $50.06 \pm 2.51$ & $58.26 \pm 0.89$ & $\mathbf{6 7 . 4 4} \pm \mathbf{1 . 7 3}$ \\
ORL & $53.78 \pm 2.56$ & $65.79 \pm 1.54$ & $64.57 \pm 1.12$ & $58.65 \pm 2.51$ & $64.26 \pm 0.89$ & $\mathbf{7 1 . 5 0} \pm \mathbf{1 . 7 3}$ \\
Yale & $35.73 \pm 2.49$ & $42.72 \pm 2.30$ & $43.77 \pm 1.58$ & $38.84 \pm 0.98$ & $42.72 \pm 1.12$ & $\mathbf{4 7 . 5 6} \pm \mathbf{1 . 1 9}$ \\
\hline
\end{tabular}

TABLE 4: Clustering performance on global noise contaminated datasets. The best performances are highlighted in bold font.

(a)

\begin{tabular}{lcccccc}
\hline \multirow{2}{*}{ Dataset } & & \multicolumn{2}{c}{ Accuracy (\%) } \\
& Kmeans & PCA & NMF & GNMF & SPNMF & RSPNMF \\
\hline FERET & $23.76 \pm 1.68$ & $27.14 \pm 1.57$ & $26.9 \pm 1.23$ & $25.76 \pm 0.58$ & $28.24 \pm 0.73$ \\
ORL & $42.36 \pm 3.24$ & $52.78 \pm 2.48$ & $52.76 \pm 2.85$ & $52.85 \pm 0.79$ & $53.91 \pm 1.78$ & $\mathbf{6 1 . 5} \pm \mathbf{1 . 9 2}$ \\
Yale & $36.79 \pm 2.24$ & $41.21 \pm 2.98$ & $42.03 \pm 2.83$ & $40.06 \pm 1.43$ & $43.64 \pm 1.39$ & $\mathbf{5 0 . 3 0} \pm \mathbf{1 . 5 7}$ \\
\hline
\end{tabular}

(b)

\begin{tabular}{lccccrr}
\hline \multirow{2}{*}{ Dataset } & & \multicolumn{4}{c}{ Normalized mutual information (\%) } \\
& Kmeans & PCA & NMF & GNMF & SPNMF & RSPNMF \\
\hline FERET & $50.06 \pm 2.74$ & $58.95 \pm 1.02$ & $57.94 \pm 1.28$ & $60.33 \pm 0.60$ & $62.01 \pm 0.57$ & $\mathbf{6 8 . 6 5} \pm \mathbf{0 . 4 3}$ \\
ORL & $60.14 \pm 2.77$ & $70.33 \pm 1.39$ & $67.37 \pm 1.94$ & $66.7 \pm 0.80$ & $70.99 \pm 0.74$ & $\mathbf{7 6 . 9 7} \pm \mathbf{0 . 9 2}$ \\
Yale & $41.68 \pm 2.27$ & $44.78 \pm 2.09$ & $46.94 \pm 2.37$ & $45.94 \pm 0.88$ & $47.68 \pm 1.08$ & $\mathbf{5 4 . 8 9} \pm \mathbf{2 . 0 8}$ \\
\hline
\end{tabular}

number. From the convergence curves, it can be observed that the convergence rate of RSPNMF is fast, and the runtime cost of RSPNMF is acceptable.

\section{Conclusion}

In this paper, we proposed a novel nonnegative matrix factorization approach named RSPNMF. RSPNMF performs the subspace learning with the nature of manifold structure preservation and robust data reconstruction. As a result, RSPNMF is more discriminating than GNMF which only considers the local structure preservation of the clean data. Experimental results on face image clustering show that RSPNMF provides a better representation in the sense of semantic subspace.

The superiority of RSPNMF arises in the following two aspects: (a) the objective function of RSPNMF uses the $\ell_{2,1}$ norm as the discrepancy measure and therefore alleviates the outlier issue that is commonly existing in image clustering. (b) The regularization term of RSPNMF incorporates the local and distant geometric information, which leads the nonnegative matrix factorization to be performed on the specific manifold. The added geometric information generally improves the clustering results.

\section{Competing Interests}

The authors declare that they have no competing interests.

\section{Acknowledgments}

This work was supported by the Natural Science Foundation of China under Grants 61333019 and 61303168.

\section{References}

[1] R. O. Duda, P. E. Hart, and D. G. Stork, Pattern Classification, Wiley-Interscience, Hoboken, NJ, USA, 2000.

[2] M. Song, D. Tao, C. Chen, J. Bu, and Y. Yang, "Color-to-gray based on chance of happening preservation," Neurocomputing, vol. 119, pp. 222-231, 2013.

[3] X. Deng, X. Liu, M. Song, J. Cheng, J. Bu, and C. Chen, "LFEME: local features with elastic manifold embedding for human action recognition," Neurocomputing, vol. 99, no. 1, pp. 144-153, 2013.

[4] D. D. Lee and H. S. Seung, "Learning the parts of objects by non-negative matrix factorization," Nature, vol. 401, no. 6755, pp. 788-791, 1999. 
[5] D. Cai, X. He, X. Wu, and J. Han, "Non-negative matrix factorization on manifold," in Proceedings of the 8th IEEE International Conference on Data Mining (ICDM '08), pp. 6372, Pisa, Italy, December 2008.

[6] D. Cai, X. He, J. Han, and T. S. Huang, "Graph regularized nonnegative matrix factorization for data representation," IEEE Transactions on Pattern Analysis and Machine Intelligence, vol. 33, no. 8, pp. 1548-1560, 2011.

[7] X. He and P. Niyogi, "Locality preserving projections," Advances in Neural Information Processing Systems, vol. 45, no. 1, pp. 186197, 2005.

[8] X. He, S. Yan, Y. Hu, P. Niyogi, and H.-J. Zhang, "Face recognition using Laplacianfaces," IEEE Transactions on Pattern Analysis and Machine Intelligence, vol. 27, no. 3, pp. 328-340, 2005.

[9] H. Liu, Z. Wu, X. Li, D. Cai, and T. S. Huang, "Constrained nonnegative matrix factorization for image representation," IEEE Transactions on Pattern Analysis and Machine Intelligence, vol. 34, no. 7, pp. 1299-1311, 2012.

[10] Z. Li, J. Liu, and H. Lu, "Structure preserving non-negative matrix factorization for dimensionality reduction," Computer Vision \& Image Understanding, vol. 117, no. 9, pp. 1175-1189, 2013.

[11] C. Hong, J. Yu, J. Li, and X. Chen, "Multi-view hypergraph learning by patch alignment framework," Neurocomputing, vol. 118, no. 11, pp. 79-86, 2013.

[12] S. E. Palmer, "Hierarchical structure in perceptual representation," Cognitive Psychology, vol. 9, no. 4, pp. 441-474, 1977.

[13] E. Wachsmuth, M. W. Oram, and D. I. Perrett, "Recognition of objects and their component parts: responses of single units in the temporal cortex of the macaque," Cerebral Cortex, vol. 4, no. 5, pp. 509-522, 1994.

[14] N. K. Logothetis and D. L. Sheinberg, "Visual object recognition," Annual Review of Neuroscience, vol. 19, pp. 577-621, 1996.

[15] W. Xu, X. Liu, and Y. Gong, "Document clustering based on non-negative matrix factorization," in Proceedings of the 26th Annual International ACM SIGIR Conference on Research and Development in Informaion Retrieval (SIGIR '03), pp. 267-273, Toronto, Canada, July 2003.

[16] V. Monga and M. K. Mihçak, "Robust and secure image hashing via non-negative matrix factorizations," IEEE Transactions on Information Forensics \& Security, vol. 2, no. 3, pp. 376-390, 2007.

[17] T. Zhang, B. Fang, Y. Y. Tang, G. He, and J. Wen, "Topology preserving non-negative matrix factorization for face recognition," IEEE Transactions on Image Processing, vol. 17, no. 4, pp. 574584, 2008.

[18] S. S. Bucak and B. Gunsel, "Video content representation by incremental non-negative matrix factorization," in Proceedings of the 14th IEEE International Conference on Image Processing (ICIP '07), pp. 113-116, San Antonio, Tex, USA, September 2007.

[19] J. B. Tenenbaum, V. De Silva, and J. C. Langford, "A global geometric framework for nonlinear dimensionality reduction," Science, vol. 290, no. 5500, pp. 2319-2323, 2000.

[20] S. T. Roweis and L. K. Saul, "Nonlinear dimensionality reduction by locally linear embedding," Science, vol. 290, no. 5500, pp. 2323-2326, 2000.

[21] M. Belkin and P. Niyogi, "Laplacian eigenmaps for dimensionality reduction and data representation," Neural Computation, vol. 15, no. 6, pp. 1373-1396, 2003.

[22] Z. Zhang and H. Zha, "Principal manifolds and nonlinear dimensionality reduction via tangent space alignment," SIAM Journal on Scientific Computing, vol. 26, no. 1, pp. 313-338, 2005.
[23] D. D. Lee and H. S. Seung, "Algorithms for non-negative matrix factorization," in Proceedings of the 14th Annual Neural Information Processing Systems Conference (NIPS '00), Denver, Colo, USA, December 2000.

[24] P. O. Hoyer, "Non-negative matrix factorization with sparseness constraints," Journal of Machine Learning Research, vol. 5, no. 12, pp. 1457-1469, 2004.

[25] J. Kim and H. Park, Sparse Nonnegative Matrix Factorization for Clustering, Georgia Institute of Technology, 2008.

[26] S. Z. Li, X. W. Hou, H. J. Zhang, and Q. S. Cheng, "Learning spatially localized, parts-based representation," in Proceedings of the IEEE Computer Society Conference on Computer Vision and Pattern Recognition (CVPR '01), pp. I-207-I-212, IEEE, Kauai, Hawaii, USA, December 2001.

[27] C. Ding, T. Li, W. Peng, and H. Park, "Orthogonal nonnegative matrix t-factorizations for clustering," in Proceedings of the 12th ACM SIGKDD International Conference on Knowledge Discovery and Data Mining (KDD '06), pp. 126-135, Philadelphia, Pa, USA, August 2006.

[28] C. Ding, T. Li, and M. I. Jordan, "Convex and semi-nonnegative matrix factorizations," IEEE Transactions on Pattern Analysis and Machine Intelligence, vol. 32, no. 1, pp. 45-55, 2010.

[29] R. Sandler and M. Lindenbaum, "Nonnegative matrix factorization with earth mover's distance metric for image analysis," IEEE Transactions on Pattern Analysis and Machine Intelligence, vol. 33, no. 8, pp. 1590-1602, 2011.

[30] N. Y. Guan, D. C. Tao, Z. G. Lu, and J. S. Taylor, "MahNMF: manhattan non-negative matrix factorization," https://arxiv.org/ abs/1207.3438.

[31] M. E. Wall, A. Rechtsteiner, and L. M. Rocha, "Singular value decomposition and principal component analysis," Physics, vol. 5, pp. 91-109, 2002.

[32] H. Wang, F. Nie, H. Huang, and F. Makedon, "Fast nonnegative matrix tri-factorization for large-scale data co-clustering," in Proceedings of the 22nd International Joint Conference on Artificial Intelligence (IJCAI '11), pp. 1553-1558, Barcelona, Spain, July 2011.

[33] S. Zafeiriou, A. Tefas, I. Buciu, and I. Pitas, "Exploiting discriminant information in nonnegative matrix factorization with application to frontal face verification," IEEE Transactions on Neural Networks, vol. 17, no. 3, pp. 683-695, 2006.

[34] J. Yang, S. Yang, Y. Fu, X. Li, and T. Huang, "Non-negative graph embedding," in Proceedings of the 26th IEEE Conference on Computer Vision and Pattern Recognition (CVPR '08), pp. 18, IEEE, Anchorage, Alaska, USA, June 2008.

[35] J. J.-Y. Wang, H. Bensmail, and X. Gao, "Multiple graph regularized nonnegative matrix factorization," Pattern Recognition, vol. 46, no. 10, pp. 2840-2847, 2013.

[36] S. An, J. Yoo, and S. Choi, "Manifold-respecting discriminant nonnegative matrix factorization," Pattern Recognition Letters, vol. 32, no. 6, pp. 832-837, 2011.

[37] N. Guan, D. Tao, Z. Luo, and B. Yuan, "Manifold regularized discriminative nonnegative matrix factorization with fast gradient descent," IEEE Transactions on Image Processing, vol. 20, no. 7, pp. 2030-2048, 2011.

[38] R. Zhi, M. Flierl, Q. Ruan, and W. B. Kleijn, “Graph-preserving sparse nonnegative matrix factorization with application to facial expression recognition," IEEE Transactions on Systems, Man, and Cybernetics, Part B: Cybernetics, vol. 41, no. 1, pp. 38$52,2011$. 
[39] Q. Gu and J. Zhou, "Co-clustering on manifolds," in Proceedings of the 15th ACM SIGKDD International Conference on Knowledge Discovery and Data Mining (KDD '09), pp. 359-367, July 2009.

[40] Q. Gu and J. Zhou, "Transductive classification via dual regularization," in Machine Learning and Knowledge Discovery in Databases, W. Buntine, M. Grobelnik, D. Mladenić, and J. Shawe-Taylor, Eds., vol. 5781 of Lecture Notes in Computer Science, pp. 439-454, 2009.

[41] D. Kong, C. Ding, and H. Huang, "Robust nonnegative matrix factorization using L21-norm," in Proceedings of the 20th ACM Conference on Information and Knowledge Management (CIKM '11), pp. 673-682, Glasgow, Scotland, October 2011.

[42] J. Huang, F. Nie, H. Huang, and C. Ding, "Robust manifold nonnegative matrix factorization," ACM Transactions on Knowledge Discovery from Data, vol. 8, no. 3, article 11, 2014.

[43] H. Zhang, Z.-J. Zha, Y. Yang, S. C. Yan, and T.-S. Chua, "Robust (semi) nonnegative graph embedding," IEEE Transactions on Image Processing, vol. 23, no. 7, pp. 2996-3012, 2014.

[44] Q. Gu, C. Ding, and J. Han, "On trivial solution and scale transfer problems in graph regularized NMF," in Proceedings of the 22nd International Joint Conference on Artificial Intelligence (IJCAI '11), pp. 1288-1293, Barcelona, Spain, July 2011. 


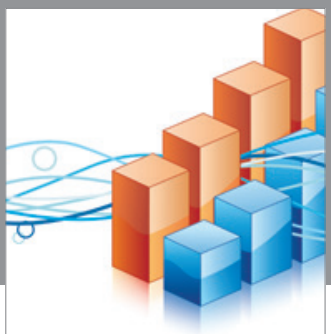

Advances in

Operations Research

vatem alat4

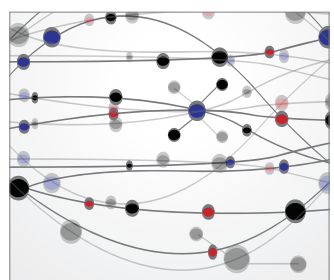

\section{The Scientific} World Journal
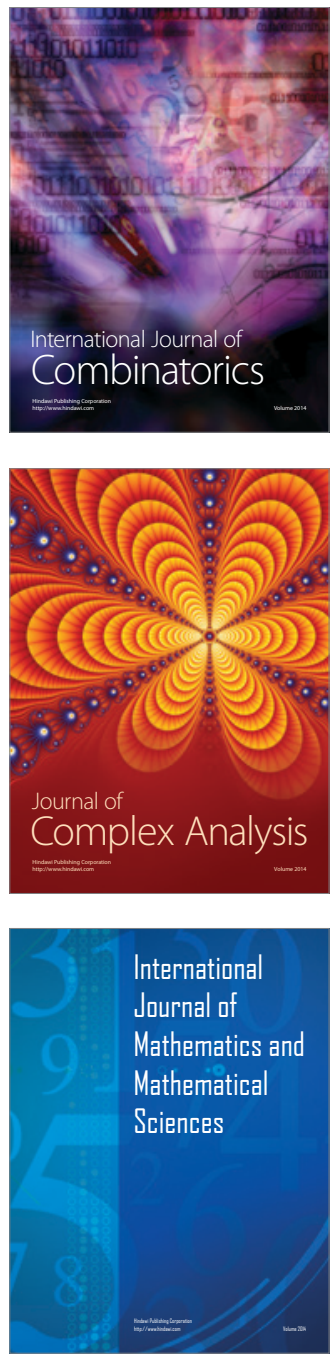
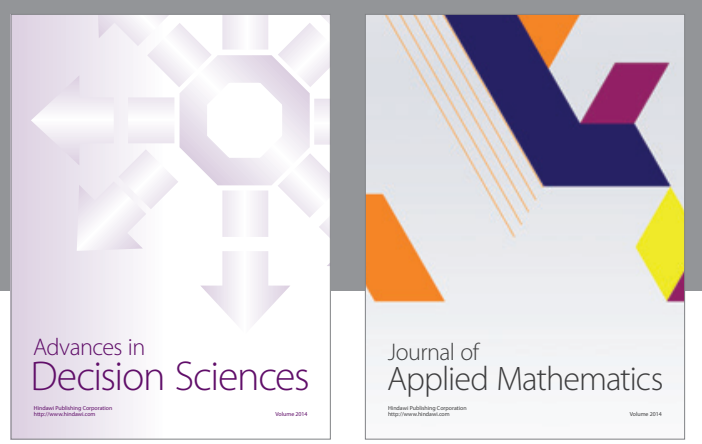

Algebra

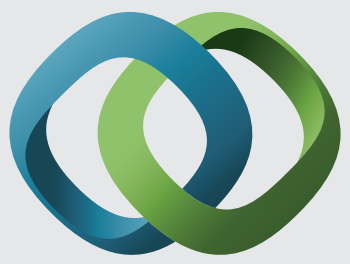

\section{Hindawi}

Submit your manuscripts at

http://www.hindawi.com
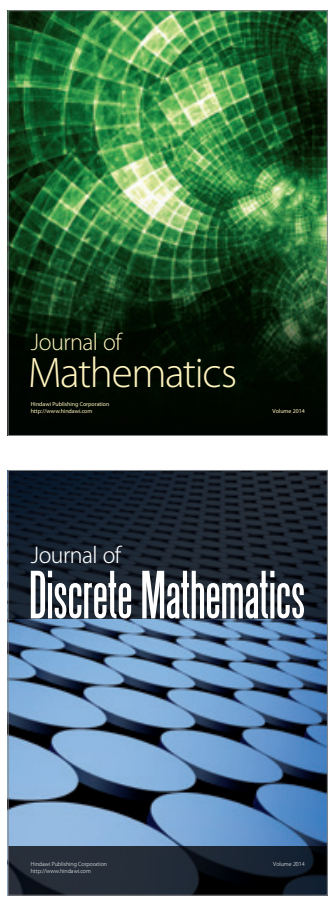

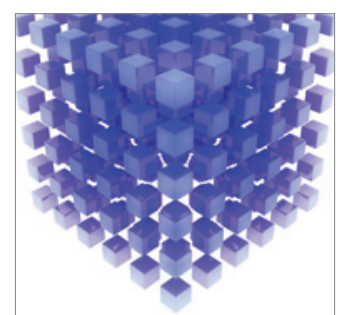

Mathematical Problems in Engineering
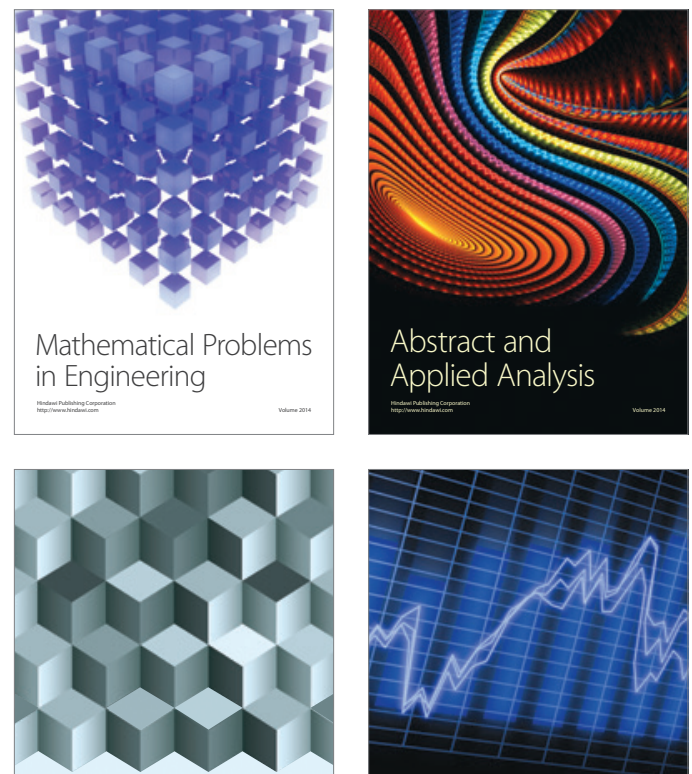

Journal of

Function Spaces

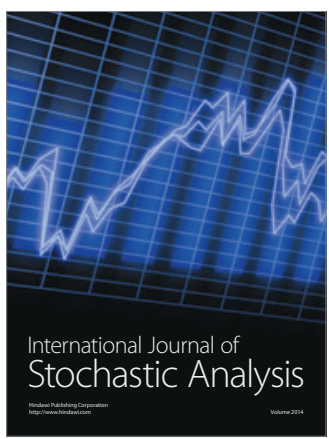

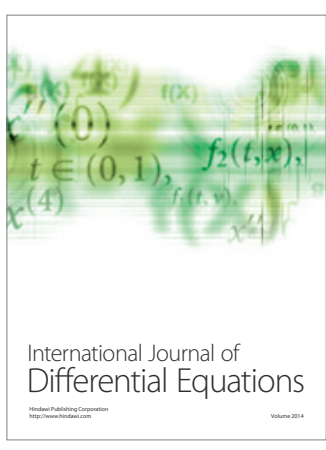
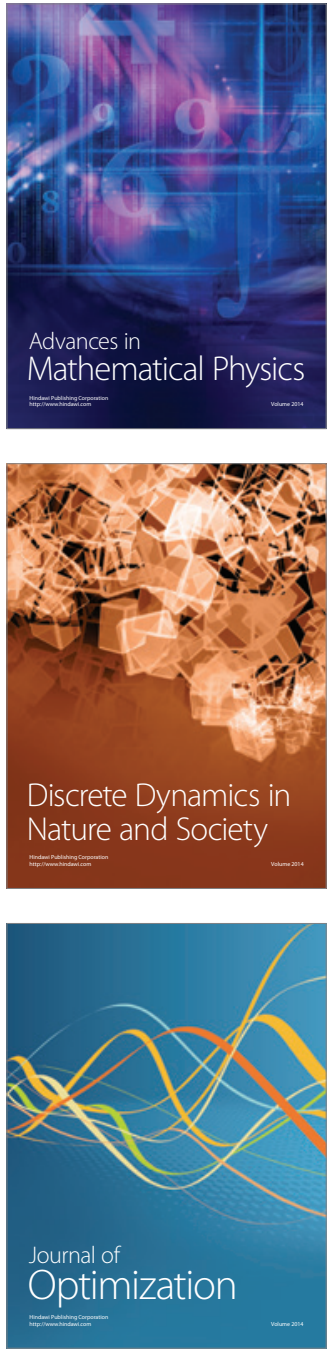